\title{
Iron Overdose during Pregnancy: Case and Treatment Review
}

\author{
Matthew J. Geraci ${ }^{*}$, Haesuk Heagney ${ }^{2}$ \\ ${ }^{1}$ Baptist Medical Center Downtown, Emergency Department, Jacksonville, Florida, USA; ${ }^{2}$ Mayo Clinic, Department of Pharmacy, \\ Jacksonville, Florida, USA. \\ Email: ${ }^{*}$ matthew.geraci@bmcjax.com
}

Received September $20^{\text {th }}, 2012$; revised October $24^{\text {th }}, 2102$; accepted November $15^{\text {th }}, 2012$

\begin{abstract}
A 22-year-old pregnant female was transferred to the emergency department having ingested a bottle of iron-containing prenatal vitamins, ondansetron $\left(\right.$ Zofran $\left.^{B}\right)$ tablets and alcohol. The patient was hemodynamically stable but suffered from intense bouts of brown, sandy emesis for the first few hours. Investigation revealed the patient ingested 13.57 $\mathrm{mg} / \mathrm{kg}$ of elemental iron. Due to the initial iron level, history and presentation time whole bowel irrigation was initiated with polyethylene glycol solution. Acute iron toxicity in pregnancy is a medical emergency that can result in multisystem organ failure leading to maternal death and potential fetal demise. High maternal serum iron loads do not affect the developing fetus and are not associated with fetal malformations; however advanced poisoning can lead to maternal death, spontaneous abortions or preterm emergency deliveries. Initial treatment strategies may include whole bowel irrigation using polyethylene glycol electrolyte lavage solution and deferoxamine treatment along with necessary supportive care management. Despite concerns of teratogenicity deferoxamine does not cross the placenta and is regarded as safe for use during pregnancy. Maternal resuscitation must always be the primary objective in acute iron overdoses and, therefore such concern should not delay clinically indicated maternal treatment.
\end{abstract}

Keywords: Iron Overdose; Pregnancy; Whole Bowel Irrigation; Deferoxamine

\section{Introduction}

A 22-year-old female was transferred from an outlying military hospital for an intentional multi-drug overdose. The patient was reportedly pregnant and had ingested a bottle of prenatal vitamins, some ondansetron $\left(\right.$ Zofran $\left.^{\circledR}\right)$ tablets along with alcohol. She presented to the military hospital a few hours after the ingestion. Per the transferring facility, the patient experienced abdominal pain, diarrhea, and vomiting prior to her arrival and during her stay. The patient's vomitous was described as tan, sandy and thick in consistency. Initial complete blood count and basic metabolic panel were unremarkable (See Table 1). The military hospital did not have the ability to perform serum iron studies and was concerned about iron toxicity secondary to excessive prenatal vitamin ingestion. Intravenous fluids and supplemental oxygen were initiated. The patient was stable with normal vital signs; the hospital transfer was uneventful, minus intermittent episodes of vomiting.

Upon transfer the patient was interviewed and labs were drawn. The patient reported multiple social issues

${ }^{*}$ Corresponding author. that caused a severe depression. She was enlisted in the Navy and had been recently relocated across the country while her boyfriend remained behind. Compounding the situation she had discovered her pregnancy the day prior. She stated she did not intend to harm or injure herself but was confused and upset. The patient admitted to ingesting 99 prenatal vitamins, ten ondansetron (Zofran $\left.{ }^{\circledR}\right) 4 \mathrm{mg}$ tablets along with a single shot of rum. She occasionally used tobacco and alcohol. There was no past history of psychiatric disorders or self harm. She was approximately 6-week pregnant, confirmed by an HCG-urine level of $37,471 \mathrm{mlU} / \mathrm{mL}$. Given the clinical picture, patient's story, and clear rapid urine toxicology screen an overdose of iron containing vitamins appeared to be the most concerning issue.

The patient's dispensing pharmacy was contacted to determine the exact type of prenatal vitamin prescribed. It was determined that each prenatal vitamin the patient had ingested contained $27 \mathrm{mg}$ of ferrous fumarate. The amount of elemental iron the patient had ingested was calculated $[1,2]$ :

$1 \mathrm{mg}$ ferrous fumarate contains $33 \%$ elemental iron.

With each tablet containing $27 \mathrm{mg}$ of ferrous fumarate: 
Table 1. Lab values.

\begin{tabular}{|c|c|c|c|c|c|c|c|c|}
\hline \multicolumn{2}{|l|}{ Day } & \multicolumn{4}{|c|}{1} & \multicolumn{2}{|c|}{2} & \multirow{2}{*}{$\begin{array}{c}3 \\
0420\end{array}$} \\
\hline Time & Ingestion - 0930 & 1440 & 1830 & 2045 & 2245 & 0420 & 1700 & \\
\hline \multicolumn{9}{|c|}{ Complete Blood Count } \\
\hline & Reference Range & & & & & & & \\
\hline White Blood Cells & $4.5-11 \mathrm{k} / \mathrm{microL}$ & 10.1 & 12 & 13.6 & & & & \\
\hline Hemoglobin & $12-16 \mathrm{gm} / \mathrm{dL}$ & 15.3 & 15.4 & 13.4 & & & & \\
\hline Hematocrit & $36 \%-46 \%$ & 45.2 & 45.6 & 38.9 & & & & \\
\hline Platelets & $150-450 \mathrm{~K} / \mathrm{microL}$ & 200 & 194 & 173 & & & & \\
\hline \multicolumn{9}{|c|}{ Basic Metabolic Panel } \\
\hline Sodium & $135-145 \mathrm{mEq} / \mathrm{L}$ & 135 & 138 & $134 \downarrow$ & & & & \\
\hline Chloride & $98-110 \mathrm{mEq} / \mathrm{L}$ & 105 & 108 & 107 & & & & \\
\hline Potassium & $3.5-5.1 \mathrm{mEq} / \mathrm{L}$ & 3.7 & 4.7 & 3.8 & & & & \\
\hline Carbon Dioxide & $22-32 \mathrm{mEq} / \mathrm{L}$ & 22 & $21 \downarrow$ & $18 \downarrow$ & & & & \\
\hline Blood Urea Nitrogen & $7-23 \mathrm{mg} / \mathrm{dL}$ & $6 \downarrow$ & $6 \downarrow$ & $4 \downarrow$ & & & & \\
\hline Serum Creatinine & $0.7-1.5 \mathrm{mg} / \mathrm{dL}$ & 0.72 & 0.88 & 0.72 & & & & \\
\hline Serum Glucose & $65-100 \mathrm{mg} / \mathrm{dL}$ & 85 & 82 & 67 & & & & \\
\hline Iron & $28-170 \mathrm{mcg} / \mathrm{dl}$ & & $236 \uparrow$ & $236 \uparrow$ & $198 \uparrow$ & 125 & 134 & 73 \\
\hline
\end{tabular}

$27 \mathrm{mg} \times 0.33=8.91 \mathrm{mg}$ elemental iron per tablet

99 tablets ingested $\times 8.91 \mathrm{mg}$ elemental iron per $\mathrm{tab}=882.09 \mathrm{mg}$ elemental iron ingested

Patient's Actual Body Weight $=65 \mathrm{~kg}$

$882.09 \mathrm{mg} / 65 \mathrm{~kg}=13.57 \mathrm{mg} / \mathrm{kg}$ elemental iron ingested

Initial serum iron concentration 9 hours after ingestion was $236 \mathrm{mcg} / \mathrm{dL}$ (Normal reference range: 28 - $170 \mathrm{mcg}$ / dl). Based on vital signs and laboratory values, including the initial serum iron concentration, whole bowel irrigation utilizing polyethylene glycol electrolyte lavage solution (PEG-ELS) was selected to enhance elimination and limit further iron absorption. Evaluation using abdominal radiograph was deferred since it was determined that the detection of iron tablets in the GI tract or a bezoar formation would not change the initial treatment strategy. Deferoxamine therapy, although reportedly safe in pregnancy, was deferred due to the dose of elemental iron, iron levels, and time of presentation [3-10].

The PEG-ELS (GoLytely ${ }^{\circledR}$ ) solution was mixed and placed in a tube feed bag. This was attached to the patient's nasogastric tube and administered via gravity at a continuous rate of $2 \mathrm{~L}$ per hour. Approximately 8 hours after initiation of whole bowel irrigation, clear effluent was observed. The patient was admitted to the hospital for medical and psychiatric management. Serum iron le- vels trended downward over the duration of her stay (See Table 1). Upon discharge she was returned to the Naval Base Hospital for further evaluation, management, and prenatal care. The patient was unable to be followed due to military transfer. See Figure 1 for timeline of patient care.

\section{Discussion}

Iron plays an extremely crucial role in normal human physiology; it is involved in oxygen transport and the regulation and differentiation of cell growth [1]. Iron supplementation is considered necessary during pregnancy. Due to increasing blood volume, fetal growth and depletion of vitamins, minerals and metabolic cofactors, diet alone may not provide enough necessary elements for proper growth. Iron containing supplements are often thought of as benign and are readily available in many forms through retail stores and prescriptions. Unfortunately, iron was reported to be one of the most widely overdosed medications in pregnant women along with analgesics (particularly acetaminophen), sedatives, antibiotics, and antihistamines [6,8]. It is important for the clinician to recognize the dangers of iron over use and to understand patient management in an overdose situation, especially in pregnancy. 


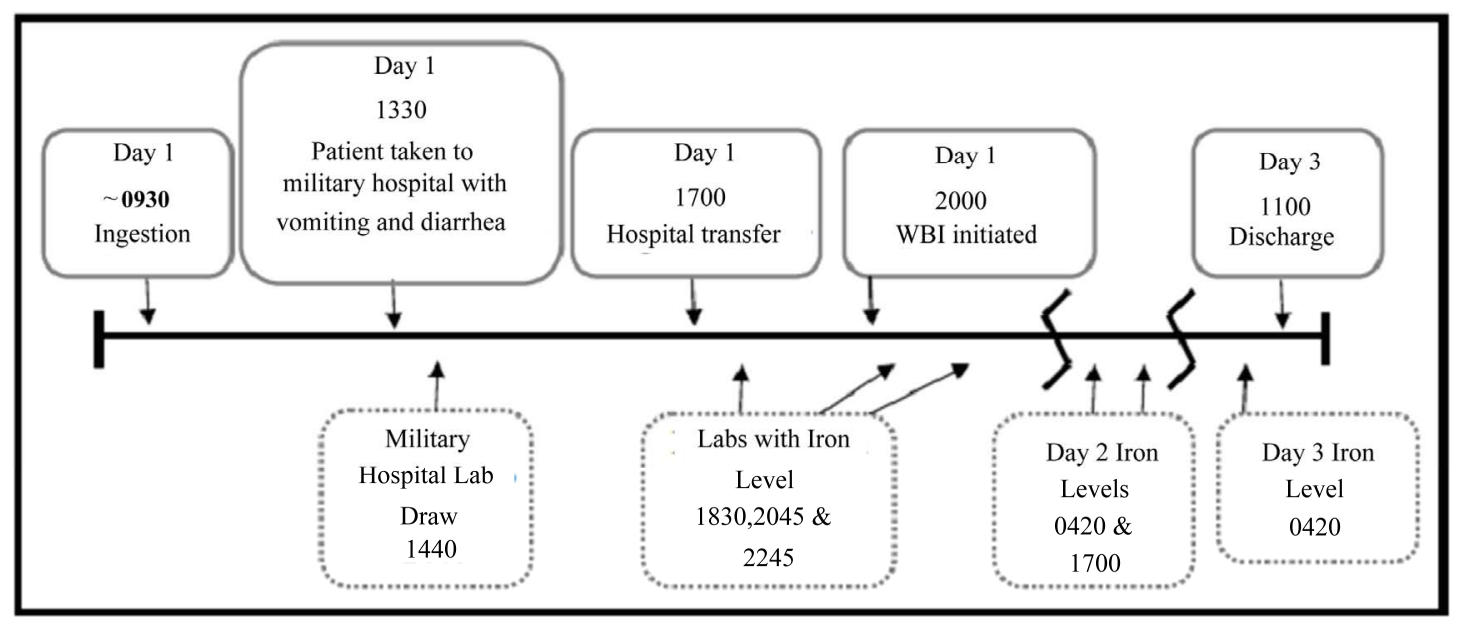

Figure 1. Timeline of ingestion.

\subsection{Pathophysiology}

As a transition metal, iron is highly involved in the reduction-oxidation reaction. Iron readily shifts from the ferric $\left(\mathrm{Fe}^{3+}\right)$ to ferrous $\left(\mathrm{Fe}^{2+}\right)$ state by accepting and donating electrons. Between the two different forms of iron, ferric and ferrous, ferrous is better absorbed in the gastrointestinal tract, particularly in the duodenum. Via transferrin (transported iron) and ferritin (stored iron), the absorption of iron is closely regulated $[1,11]$.

In an overdose situation, initial symptoms and tissue damage occur in the gastrointestinal epithelium. Systemic toxicity begins once iron-induced reactive oxygen species enters the circulation. When serum transferrin becomes saturated, "free" iron circulates to various organ systems and promotes oxidative damage. Elevated iron concentrations can be detected in the stomach, liver, brain, heart, lung, small bowel, and kidney. These findings are consistent with presentations observed in patients with severe iron toxicity. In addition, iron toxicity can lead to intracellular damage causing disruption of mitochondrial oxidative phosphorylation. This may promote a shift towards anaerobic metabolism placing the patient in a state of metabolic acidosis which may further contribute to harm. Iron toxicity can also cause excessive post arteriolar dilation, increased capillary permeability, and coagulopathy which may lead to severe acidosis and shock [4,8,11-15].

In pregnancy, the fetus does not seem susceptible to high maternal serum iron loads; nor have fetal malformations been associated with iron overdoses. Animal and human data demonstrate no increased iron loads in fetal circulation or in umbilical cord blood [8]. Maternal health and treatment is paramount in managing these cases.

\subsection{Clinical Manifestations}

Clinical manifestations of iron toxicity can be divided into five different stages and is associated with the timing of iron ingestion as well as presenting symptoms (See Table 2). This is highly dependent on the pathophysiology of iron toxicity as mentioned above $[8,11]$.

Initial gastrointestinal symptoms such as abdominal pain, nausea, vomiting, and diarrhea will be common. Severe overdoses may present with early and significant hemodynamic instability. The second stage of iron toxicity involves metabolic acidosis as a result of ongoing cellular damage. While gastrointestinal symptoms may resolve, patients will likely become lethargic, hypotensive, and tachycardic. According to Lacoste et al., high serum iron concentration (i.e. greater than $300 \mathrm{mcg} / \mathrm{dL}$ ) is commonly associated with a white blood cell count higher than 15,000 cells/uL or plasma glucose greater than $150 \mathrm{mg} / \mathrm{dL}$ [5]. The third stage represents worsening hemodynamic instability and death may ensue should the patient present for care late or if the iron toxicity is inadequately managed. The fourth stage involves direct cytotoxicity to various organ systems. As severe iron toxicity may cause wide distribution of iron to nearly all organ systems, hepatic toxicity, renal toxicity, and even cardiac toxicity can be observed. Failure to appropriately respond to acute iron toxicity may lead to death [8-11].

\section{Evaluation}

A thorough history should be ascertained when evaluating iron toxicity. The time of ingestion, form of iron supplement ingested, the amount of elemental iron ingested, and the start time of gastrointestinal symptoms should be identified in order to determine the potential severity of toxicity as well as treatment strategies. It is important to know the exact form of iron ingested to assist with evaluation. The three most common forms of iron in prenatal vitamin supplements include ferrous fumarate, ferrous sulfate and ferrous gluconate; containing $33 \%, 20 \%$, and $12 \%$ elemental iron respectively. Labo- 
Table 2. Clinical manifestations.

\begin{tabular}{|c|c|}
\hline Stages & Time line and associated symptoms \\
\hline 1 & $\begin{array}{c}1 \text { - } 6 \text { hours after ingestion: Nausea, vomiting, abdominal pain, diarrhea Intestinal ulceration, edema, small-bowel infarction and } \\
\text { necrosis }\end{array}$ \\
\hline 2 & 6 - 24 hours after ingestion: Resolution of GI symptoms lethargy, tachycardia, hypotension, metabolic acidosis \\
\hline 3 & 12 - 24 hours after ingestion: Shock from hypovolemia, vasodilation, poor cardiac output, hyperventilation, seizures, coma \\
\hline 4 & 2 - 3 days after ingestion: Hepatotoxicity, renal failure, cardiac failure \\
\hline 5 & 2 - 8 weeks after ingestion: Gastric outlet obstruction secondary to strictures and scarring \\
\hline
\end{tabular}

ratory values including complete blood count, basic metabolic panel, liver panel, coagulation panel, serum iron concentration, should be monitored.

Literature suggests that $10-20 \mathrm{mg} / \mathrm{kg}$ of ingested elemental iron can cause mild toxicity resulting in gastrointestinal symptoms such as abdominal pain, nausea, vomiting, and diarrhea. Elemental iron ingestions of 40 $\mathrm{mg} / \mathrm{kg}$ or greater is considered severe toxicity. In addition, serum iron concentration of $300 \mathrm{mcg} / \mathrm{dL}$ or above can present as moderate toxicity. Serum iron concentration of $500 \mathrm{mcg} / \mathrm{dL}$ or above represents severe toxicity and above $1000 \mathrm{mcg} / \mathrm{dL}$ can be lethal. Because ingested iron may continue to be absorbed as it transits the duodenum and upper jejunum, immediate initiation of detoxification therapy is critical even if initial numbers do not represent severe toxicity. Laboratory values and changes in patient presentation should guide decision-making and treatment options $[8,11,13]$.

\section{Treatment Overview}

Pregnancy should not delay or preclude therapy when an iron overdose is suspected. Maternal danger and toxicity should receive immediate evaluation, studies, and therapy. Fears of potential teratogenicity should not interrupt initiation of such treatment, as fetal safety is dependant on maternal health.

Presentations may vary widely as symptoms may differ based on time of ingestion, amount and type of iron pills ingested, as well as if there were any significant coingestants involved in the situation. The patient will likely require intravenous volume repletion and supplemental oxygen. If necessary, patient may require intubation to keep the airway patent and prevent aspiration. Results of laboratory values such as arterial blood gases, complete blood counts, metabolic panels, coagulation panel, and hepatic profile should further assist in developing supportive care strategies.

\subsection{Abdominal Radiograph}

Abdominal radiograph may be considered for the purpose of visualizing radio opaque iron tablets [11]. Radio opacity may vary by form of iron supplement ingested, time since ingestion, and amount of elemental iron ingested. Oral iron formulations can conglomerate in the gut to form a solid mass or bezoar $[11,13]$. Such formation can further complicate the toxicity as it can serve as a reservoir for iron and result in variable, continuous iron release. Radiographic findings may be particularly helpful in guiding and evaluating the success of treatment.

Should the clinician believe an abdominal radiograph is warranted, pregnancy should not preclude this evaluation. The American College of Radiology states that pregnancy is a relative contraindication to ionizing imaging modalities. A single diagnostic X-ray procedure does not result in a radiation dose adequate to threaten the wellbeing of the developing pre-embryo, embryo, or fetus. The American College of Obstetricians and Gynecologists agrees with this position [16-19].

\subsection{Gastrointestinal Decontamination}

Upon stabilization of the patient, gastrointestinal decontamination strategies should be considered to promote excretion and limit further iron absorption. Although several methods may be employed, whole bowel irrigation (WBI) has consistently shown efficacy against iron ingestions [11,20-23].

Gastrointestinal decontamination strategies used for iron toxicity have previously included induced emesis, activated charcoal, and orogastric lavage. In the case of iron toxicity, these strategies have been proven ineffective and some are considered unsafe. Literature published prior to the 1980's supported the use of syrup of ipecac for induction of emesis. However, ipecac use has fallen out of favor. The clinical benefit of ipecac is not worth the risks of aspiration and possible esophageal damage that can be caused from violent, continuous episodes of chemically induced emesis. Syrup of ipecac use can also cloud the clinical picture, as vomiting is a symptom and one of the early signs of a toxic iron ingestion. Unlike many toxicology cases, use of activated charcoal is limited in iron ingestions. Activated charcoal does not significantly bind or adsorb iron. The use of orogastric lavage is also limited due to the large size and poor solubility of most iron tablets. Orogastric lavage may not be effective once the bulk of the iron tablets move past the 
pylorus. Gastric lavage with bicarbonate has led to some systemic toxicity as well and is not routinely recommended. Should orogastric lavage be selected as a management strategy for iron toxicity, only a $0.9 \%$ sodium chloride solution or tap water should be utilized $[8,11$, 13].

WBI is an effective, safe, and non-invasive gastrointestinal decontamination modality for management of iron toxicity. Van Ameyde et al. have reported a successful management of iron toxicity in a pregnant woman during her third trimester [23]. Using an osmotically balanced polyethylene glycol electrolyte lavage solution (PEG-ELS), WBI prevents further iron absorption by inducing a liquid stool. Because PEG solution contains balanced electrolytes, WBI with PEG can result in resolution of electrolyte imbalance. Various PEG-ELS formulations are available and may differ slightly in electrolyte content [20-24].

The usual dose of PEG-ELS is 1.5 - $2 \mathrm{~L} /$ hour for adults and $500 \mathrm{~mL} /$ hour for children. Prior to initiation, a bedside commode should be in place. It is crucial that administration via a nasogastric tube is accomplished by starting slowly and increasing its rate as tolerated. Furthermore, such administration should be continued until rectal effluent is clear and iron levels reliably trend downward. Iron bezoar formation and adhesion to the GI mucosal lining may complicate this therapy. Kaczorowski et al. report a successful five days of continuous whole bowel irrigation with a total of $44.3 \mathrm{~L}$ of PEG- ELS to overcome iron retention in the gut of a child [22]. The goal of WBI is to remove as much of the iron as possible as to avoid more invasive measures. Iron may form stubborn masses or bezoars that adhere to and can penetrate through the entire thickness of gastrointestinal walls. Endoscopy, laparotomy and gastrotomy procedures may become an option to remove iron aggregates that are refractory to other methods $[25,26]$.

Several possible adverse effects of WBI include vomiting, abdominal bloating, fullness, cramping, flatulence, and pruritus ani. It may also exacerbate congestive heart failure in an unstable patient with cardiac and renal dysfunction [21]. Contraindications for WBI may include patients with unprotected or compromised airway, bowel obstruction, ileus or perforation, clinically significant gastrointestinal bleed, intractable vomiting, and unstable vital signs [21].

\subsection{Deferoxamine}

Deferoxamine is a chelating agent that exhibits high affinity and specificity for iron, does not cross the placenta and is safe for use in pregnancy [8]. It has been found that deferoxamine easily chelates iron from ferritin, hemosiderin, and transferrin. Conversely deferoxamine does not bind or remove iron from hemoglobin or the cyto- chrome enzymes. Deferoxamine may chelate intracellular extramitochondrial iron.

Deferoxamine preferentially forms complexes with the ferric iron $\left(\mathrm{Fe}^{3+}\right)$. Approximately $1000 \mathrm{mg}$ of deferoxamine can bind $85 \mathrm{mg}$ of ferric iron. When this complex is formed it is known as ferrioxamine. Ferrioxamine is excreted via the kidneys and such excretion can be observed via a characteristic vin-rose coloring of the urine. Such observation may indicate efficacy of deferoxamine but cannot be the sole determinant of reduced iron levels $[11,27,28]$.

There are several presentations that may necessitate immediate initiation of deferoxamine therapy. They are: presence of metabolic acidosis, repetitive vomiting, lethargy, hypotension, or signs of shock. If the serum iron concentration is greater than $400 \mathrm{mcg} / \mathrm{dL}$ or if more than $20 \mathrm{mg} / \mathrm{kg}$ elemental iron is ingested, deferoxamine therapy should be initiated $[8,11,13]$.

Once maternal indication for deferoxamine therapy is confirmed, an initial dose of $1000 \mathrm{mg}$ should be administered IV or IM. It is crucial that IV deferoxamine is administered at a rate not exceeding $15 \mathrm{mg} / \mathrm{kg} / \mathrm{hour}$ to avoid infusion related reactions. This first $1000 \mathrm{mg}$ of deferoxamine may be followed by two doses of $500 \mathrm{mg}$ at 4-hour intervals. An additional $500 \mathrm{mg}$ may be administered over 4 - 12 hours; the total amount administered should not exceed $6000 \mathrm{mg}$ in 24 hours. Subsequent doses should not exceed a rate of $125 \mathrm{mg} /$ hour [28]. The same dosing schedule will apply if intramuscular administration is selected.

To avoid flushing of the skin, urticaria, hypotension, and shock from rapid administration, deferoxamine should be given intramuscularly or via slow intravenous administration. The manufacturer recommends intramuscular administration for all patients not in shock and intravenous administration for patients in a state of cardiovascular compromise. However, literature supports the use of slow intravenous administration regardless of the patient's state. Subcutaneous administration is not recommended for the treatment of acute iron toxicity $[3,7,28]$.

There have been reports of allergic-type reactions including cutaneous wheal formation, pruritus, rash, and anaphylactoid reactions from long-term utilization of deferoxamine. Neurologic, respiratory, ocular, and otic adverse effects have been reported as well [28]. Although the mechanism is unknown, deferoxamine therapy may increase susceptibility to infection, particularly Yersinia enterocolitica and Yersinia pseudotuberculosis [11]. These adverse effects have been reported with long-term use of deferoxamine rather than short-term use like in cases of acute iron toxicity. This side effect profile should not deter healthcare professionals to choose this treatment modality.

The package insert discourages the use of deferoxami- 
ne in pregnancy. This is due to animal studies that demonstrated delayed ossification and skeletal anomalies in two animal species. However, medical literature and clinical experience in humans demonstrates success and supports utilization of deferoxamine in pregnancy. Vaskaridou et al. presented two case reports of successful deferoxamine treatment during the first trimester [10]. McElhatton et al. revealed that $8 \%$ of pregnant patients who received deferoxamine treatment delivered malformed babies. Nonetheless, deferoxamine was excluded as a cause for such result [29]. Based on the setting of transfusion dependent beta-thalassemia, Bailey reported that there have been 24 cases of normal outcomes despite receiving deferoxamine during pregnancy [30]. Singer et al. presented a successful deferoxamine treatment due to iron overload secondary to beta-thalassemia; this resulted in a birth of a healthy baby boy [7]. Deferoxamine treatment has demonstrated safety in pregnancy but is an additional therapy that should be incorporated with maintenance of a clear airway, control of hemodynamic instability, and whole bowel irrigation.

\section{Conclusion}

Acute iron toxicity in pregnancy is a medical emergency that can result in multi-system organ failure leading to maternal death and potential fetal demise. A thorough evaluation of the patient including time of ingestion, form of iron supplement ingested, and time of symptom development are important. Calculating the amount of elemental iron ingested is also critical in determining treatment strategies. High maternal serum iron loads do not affect the developing fetus and are not associated with fetal malformations; however advanced poisoning can lead to maternal death, spontaneous abortions or preterm emergency deliveries. Initial treatment strategies may include supportive care management and the removal of iron from the body using whole bowel irrigation with PEG-ELS. Deferoxamine treatment along and potential surgical interventions are also modalities that may be employed in more serious cases. Despite concerns of teratogenicity deferoxamine does not cross the placenta and is regarded as safe for use during pregnancy. Maternal resuscitation must always be the primary objective in acute iron overdoses and, therefore such concern should not delay clinically indicated maternal treatment.

\section{REFERENCES}

[1] NIH Office of Dietary Supplements, "Iron: Dietary Supplement Fact Sheet," 2007. http://ods.od.nih.gov/factsheets/iron/

[2] “Major Pharmaceuticals. Product Catalog," 2011. http://www.majorpharmaceuticals.com/

[3] P. Blanc, D. Hryhorczuk and I. Danel. "Deferoxamine Treat- ment of Acute Iron Intoxication in Pregnancy," Obstetrics \& Gynecology, Vol. 64, Suppl. 3, 1984, pp. 12S-14S.

[4] S. Khoury, M. Odeh and M. Oettinger. "Deferoxamine Treatment for Acute Iron Intoxication in Pregnancy," Acta Obstetricia et Gynecologica Scandinavica, Vol. 74, No. 9, 1995, pp. 756-757. doi:10.3109/00016349509021190

[5] H. Lacoste, G. L. Goyert, L. S. Goldman, et al., "Acute Iron Intoxication in Pregnancy: Case Report and Review of the Literature," Obstetrics \& Gynecology, Vol. 80, No. 3, 1992, pp. 500-501.

[6] W. Rayburn, R. Aronow, B. DeLancey and M. J. Hogan, "Drug Overdose during Pregnancy: An Overview from a Metropolitan Poison Control Center," Obstetrics \& Gynecology, Vol. 64, No. 5, 1984, pp. 611-614.

doi:10.1002/(SICI)1096-8652(199901)60:1<24::AID-AJ H5>3.0.CO;2-C

[7] S. T. Singer and E. P. Vichinsky, "Deferoxamine Treatment during Pregnancy: Is It Harmful?" American Journal of Hematology, Vol. 60, No. 1, 1999, pp. 24-26.

[8] T. Tran, J. R. Wax, C. Philput, et al., "Intentional Iron Overdose in Pregnancy-Management and Outcome," Journal of Emergency Medicine, Vol. 18, No. 2, 2000, pp. 225-228. doi:10.1016/S0736-4679(99)00199-7

[9] T. Tran, J. R. Wax, J. D. Steinfeld and C. J. Ingardia, "Acute Intentional Iron Overdose in Pregnancy," Obstetrics \& Gynecology, Vol. 92, No. 2, 1998, pp. 678-680.

[10] E. Vaskaridou, K. Konstantopoulos, D. Kyriakou and D. Loukopoulos, "Deferoxamine Treatment during Early Pregnancy: Absence of Teratogenicity in Two Cases," Haematologica, Vol. 78, No. 3, 1993, pp. 183-184.

[11] N. E. Flomenbaum, L. R. Goldfrank, et al., "Goldfrank's Toxicologic Emergencies," 8th Edition, McGraw-Hill, New York, 2006.

[12] A. K. Baranwal and S. C. Singhi, "Acute Iron Poisoning: Management Guidelines," Indian Pediatrics, Vol. 40, No. 6, 2003, pp. 534-540.

[13] T. Madiwale and E. Liebelt, "Iron: Not a Benign Therapeutic Drug," Current Opinion in Pediatrics, Vol. 8, No. 2, 2006, pp. 174-179. doi:10.1097/01.mop.0000193275.62366.98

[14] J. P. Pestaner, K. G. Ishak, F. G. Mullick and J. A. Centeno, "Ferrous Sulfate Toxicity a Review of Autopsy Findings," Biological Trace Element Research, Vol. 69, No. 3, 1999, pp. 191-198.

[15] C. Spanierman and A. Tarabar, "Iron Toxicity in Emergency Medicine," 2011. http://emedicine.medscape.com/article/815213-overview

[16] The American College of Obstetricians and Gynecologists, "Planning Your Pregnancy and Birth, Education Pamphlet," 3rd Edition, Washington DC. 2000. http://www.acog.org/publications/patient_education/ab01 2.cfm

[17] The American College of Obstetricians and Gynecologists, "Guidelines for Perinatal Care," 6th Edition, Washington DC, 2007.

[18] The American College of Radiology, "Practice Guideline for Imaging Pregnant or Potentially Pregnant Adolescents and Women with Ionizing Radiation," 2008. 
http://www.acr.org/SecondaryMainMenuCategories/ quality_safety/guidelines/dx/Pregnancy.aspx

[19] J. E. Gray, "Safety (Risk) of Diagnostic Radiology Exposures," In: M. L. Janower and O. W. Linton, Eds., Radiation Risk: A Primer, American College of Radiology, Reston (ACR), Reston, 1996, pp. 15-17.

[20] G. W. Everson, E. J. Bertaccini and J. O'Leary, "Use of Whole Bowel Irrigation in an Infant Following Iron Overdose," American Journal of Emergency Medicine, Vol. 9, No. 4, 1991, pp. 366-369. doi:10.1016/0735-6757(91)90060-W

[21] M. A. Howland, "Antidotes in Depth (A3): Whole-Bowel Irrigation and Other Intestinal Evacuants," In: L. S. Nelson, N. A. Lewin, M. A. Howland, et al., Eds. Goldfrank's Toxicologic Emergencies, 9th Edition, McGrawHill, New York, 2011.

http://www.accesspharmacy.com.lp.hscl.ufl.edu/content.a spx?aID $=6535269$

[22] J. M. Kaczorowski and P. M. Wax, "Five Days of WholeBowel Irrigation in a Case of Pediatric Iron Ingestion," Annals of Emergency Medicine, Vol. 27, No. 2, 1996, pp. 258-263. doi:10.1016/S0196-0644(96)70334-7

[23] K. J. Van Ameyde and M. Tenenbein, "Whole Bowel Irrigation during Pregnancy," American Journal of Obstetrics \& Gynecology, Vol. 160, No. 3, 1989, pp. 646-647.

[24] S. C. Wong and R. Kulkarni, "Whole-Bowel Irrigation," 2009. http://emedicine.medscape.com/article/1413446-print

[25] F. Haider, C. De Carli, S. Dhanani and B. Sweeney, "Emergency Laparoscopic-Assisted Gastrotomy for the Treatment of an Iron Bezoar," Journal of Laparoendoscopic \& Advanced Surgical Techniques, Vol. 19, Suppl. 1, 2009, pp. S141-S143. doi:10.1089/lap.2008.0122.supp

[26] C. D. Peterson and G. C. Fifield, "Emergency Gastrotomy for Acute Iron Poisoning," Annals of Emergency Medicine, Vol. 9, No. 5, 1980, pp. 262-264. doi:10.1016/S0196-0644(80)80385-4

[27] "Deferoxamine. Gold Standard, Clinical Pharmacology," 2011.

http://www.goldstandard.com/product/drug-reference-pati ent-education/clinical-pharmacology/ http://www.clinicalpharmacology.com/

[28] Hospira, Inc., "Deferoxamine Mesylate," Hospira, Inc., Lake Forest, 2009.

[29] P. R. McElhatton, J. C. Roberts and F. M. Sullivan, "The Consequences of Iron Overdose and Its Treatment with Desferrioxamine in Pregnancy," Human \& Experimental Toxicology, Vol. 10, No. 4, 1991, pp. 251-259. doi:10.1177/096032719101000404

[30] B. Bailey, "Are There Teratogenic Risks Associated with Antidotes Used in the Acute Management of Poisoned Pregnant Women?" Birth Defects Research Part A: Clinical and Molecular Teratology, Vol. 67, No. 2, 2003, pp. 133-140. doi:10.1002/bdra.10007 